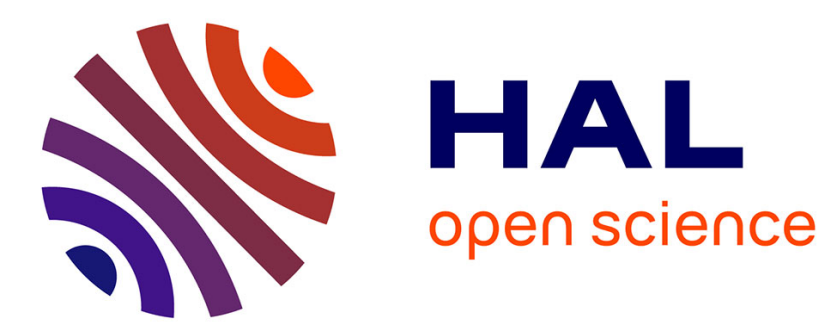

\title{
Scale invariant images in astronomy through the lens of multifractal modeling
}

Pierre Chainais, Véronique Delouille, Jean-François Hochedez

\section{To cite this version:}

Pierre Chainais, Véronique Delouille, Jean-François Hochedez. Scale invariant images in astronomy through the lens of multifractal modeling. 18th IEEE International Conference on Image Processing (ICIP2011),, Sep 2011, Brussels, Belgium. pp.1309 -1312, 10.1109/ICIP.2011.6115676 . hal-00657779

\section{HAL Id: hal-00657779 https://hal.science/hal-00657779}

Submitted on 9 Jan 2012

HAL is a multi-disciplinary open access archive for the deposit and dissemination of scientific research documents, whether they are published or not. The documents may come from teaching and research institutions in France or abroad, or from public or private research centers.
L'archive ouverte pluridisciplinaire HAL, est destinée au dépôt et à la diffusion de documents scientifiques de niveau recherche, publiés ou non, émanant des établissements d'enseignement et de recherche français ou étrangers, des laboratoires publics ou privés. 


\section{SCALE INVARIANT IMAGES IN ASTRONOMY THROUGH THE LENS OF MULTIFRACTAL MODELING}

\author{
P. Chainais $^{(1) *}$ \\ (1) Clermont Université, Univ. Blaise Pascal \\ INRIA Lille-Nord Europe (SEQUEL) \\ 59650 Villeneuve d'Ascq, France
}

\author{
V. Delouille $e^{(2) \dagger}$, J.-F. Hochedez $z^{(2,3)}$ \\ ${ }^{(2)}$ Royal Obs. of Belgium, B-1180 Brussels \\ (3) LATMOS UMR CNRS 8190, \\ 78280 Guyancourt, France
}

\begin{abstract}
The mystery of the dynamics of the Sun is even deeper when it is quiet, i.e. when and where there are no coherent areas structured by strong magnetic field and called active regions. The corona that is part of the solar atmosphere is observed by extreme UV spatial telescopes for which higher resolutions are always desired: the best pixel resolution currently achievable is $500 \mathrm{~km}$ which still leaves much room for crucial hidden details. We present our work on the statistical analysis of quiet Sun images of the corona. These images exhibit multifractal properties and a model based on scale invariant stochastic processes, called fractionally integrated compound Poisson cascades, is able to reproduce their main statistical properties. We show that it can be used to develop a virtual super-resolution method that proposes plausible predictions of the high resolution information hidden below the pixel size of present observations. Such images may help physicists to test and calibrate on-board processing algorithms (compression, detection of events) for future missions.
\end{abstract}

Index Terms - self-similarity, multifractal analysis, stochastic processes, extrapolation, solar corona

\section{INTRODUCTION}

The solar corona is a large, thermally conducting plasma heated to millions of degrees while the temperature at the surface of the Sun (the photosphere) is only of a few thousand degrees. One of the long-standing problems in solar physics is the understanding of the mechanism that heats the corona. To convert ordered energy (e.g. waves or electrical currents) into dissipated heat, unobservable small scales must somehow be produced. Hence observations at ever higher resolution are necessary. Several telescopes of increased spatial and temporal resolution have been launched since 1996,

\footnotetext{
${ }^{*}$ The first author performed the work while at LIMOS UMR CNRS 6158, Clermont Université, France. This work was supported by an Egide Tournesol grant 2007-2009.

${ }^{\dagger}$ The second author is supported by ESA/PRODEX program funded by the Belgian Federal Science Policy Office (BELSPO).
}

and more instruments are in preparation. In the quiet Sun images of the corona, scale-invariance has been observed down to the Point-Spread Function (PSF) of today's highest resolution instruments. Physical models suggest that this property should persist at least down to the scale of $100 \mathrm{~km}$ [1]. This scale invariance can be characterized using the multiscaling exponents. We have shown that a multifractal model involving compound Poisson cascades is able to generate images having the same multifractal parameters as the original observations [2]. Other approaches involving multifractal analysis were presented to study coherent magnetic structures (active regions) [3]. Our goal is to produce magnified images of the quiet Sun by inserting plausible small scales into a given observation. The plausibility stems from the statistical and physical consistency with the original observation. The 'virtually super-resolved' (VSR) images [4] will help the physicists to calibrate and test algorithms on forthcoming higher resolution instruments. It also allows to predict histograms of future high resolution imagers. For example, VSR shows that images of the quiet corona are sufficiently smooth for the histogram to be preserved even when we observe closer to the Sun, which was not a priori guaranteed. These images help in testing and choosing the best denoising method. When adapted on the 3D sphere [5] they can be used to calibrate a stereographic reconstruction algorithm. This contribution summarizes a set of works carried out over the last 5 years from the first observation of the multiscaling properties of quiet Sun coronal images to the design of our VSR method and its applications.

\section{QUIET SUN IMAGES ARE MULTIFRACTAL}

\subsection{Data set description}

Our study is based on full-Sun images taken between 2 Jan. and 28 Dec. 1997 by EIT [6] onboard SoHO in the Extreme Ultra-Violet (EUV) Fe XII line at $19.5 \mathrm{~nm}$ which corresponds to temperatures up to 1.5 millions degrees. A pixel of EIT images represents a surface of $(1800 \mathrm{~km})^{2}$ on the Sun. We 
selected a first set of $1024 \times 1024$ full Sun images recorded with at least five days spacing to ensure statistical independence between the images (rotation period at the equator $=25$ days). Further, in order to limit the shortening effect due to the curvature at the limb to roughly $15 \%$, we do not consider the full Sun images but rather disks of a half solar radius wide centered on the Sun, fig. 1(a). We have excluded images featuring active regions. We worked with a set of 54 disks centered on the Sun, all having the same field of view.

\subsection{Multifractal properties of quiet Sun images}

Guided by previous work on quiet Sun images [7, 8] and by the visual aspect of EIT images, we performed a precise study of their scale invariance properties. For isotropic images, a first criterion is given by the power-law behavior of the Fourier spectrum averaged over orientations. This necessary property is observed on quiet Sun images [7] as well as in natural images [9]. At the present resolution, the solar topology displays an isotropic scale invariance. Yet, magnetic fields should make the observation depart from isotropy at some scale related to the plasma beta, i.e. the magneticto-thermal pressure ratio. Our assumption of scale invariance would then not hold anymore. To reach order statistics larger than two, we performed a wavelet based multifractal analysis. We found reasonable multiscaling power-law behaviors of structure functions $S(q, j)$ computed as empirical moments of order $q$ of the Daubechies' wavelet coefficient modulus $[2,10]$ :

$$
S(q, j)=\frac{1}{n_{j}} \sum_{l=1}^{n_{j}}|d(j, l)|^{q} \sim 2^{j \zeta(q)}
$$

where $n_{j}$ is the number of wavelet coefficients $d(j, l)$ at scale $a=2^{j}$. The structure function $S(q, j)$ of a multifractal process obeys a powerlaw scaling behavior $S(q, j) \sim a^{\zeta(q)} \sim$ $2^{j \zeta(q)}$, at least for some range of scales $a$ and orders $q$ 's. Such an analysis reveals whether the images are simply self-similar and characterized by the single Hurst parameter $H$ such that $\zeta(q)=q H$, or multifractal and characterized by a set of exponents $\zeta(q)$. Results show unambiguously that quiet Sun images are multifractal, fig. 1(b), with $\zeta(q)=q H+\tau(q)$ where $\zeta(1)=H \simeq 0.6 \pm 0.05$ and $\tau(q)$ captures the non-linear part of $\zeta(q)$ with $\tau(0)=\tau(1)=0$. The estimated multiscaling exponents $\zeta(q)$ will be used as parameters to identify a family of stochastic processes that obey the same statistical property with the same set of parameters.

\section{MULTIFRACTAL PROCESSES TO MODEL QUIET SUN IMAGES}

\subsection{The approach}

Our purpose is to virtually refine the resolution of scale invariant textured images thanks to a stochastic augmentation

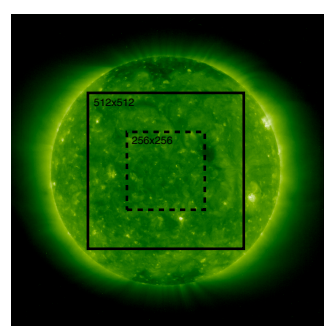

(a)

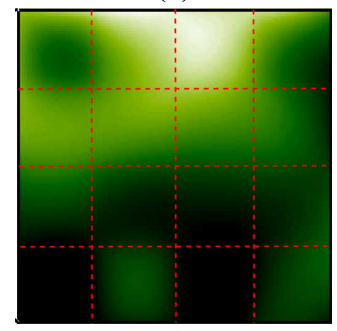

(c)

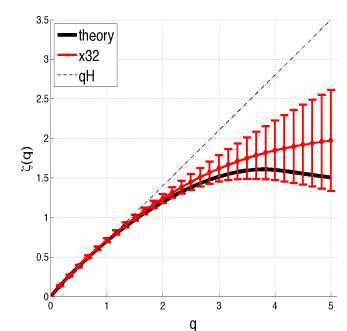

(b)

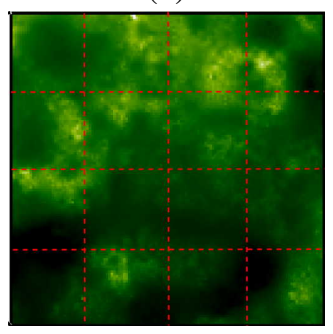

(d)
Fig. 1. (a) A quiet Sun image and the analyzed squared area. (b) Multiscaling exponents $\zeta(q)$ for quiet Sun images and for simulated images. (c) spline interpolation of a 4x4 area. (d) super-resolved version of the same area.

of information. The method is thus designed to work on initial images which have some random texture looking aspect, such as images taken from a turbulent physical system. An appropriate model should be able to reproduce and extrapolate the statistical scale invariance observed in the data (statistical consistency). It should as well preserve the light energy flux so that physical consistency be ensured. It means that degrading the resolution of super-resolved images must give the initial image back. Our modeling uses thus only minimal prior information on fundamental properties. The main prior of our approach is the assumption that same scale invariance will continue to prevail at scales yet unobserved. In the case of images from the solar corona, it is not clear down to which scale the scale-invariance observed on EIT image will continue to prevail; there is however a consensus on the assumption that the turbulent cascading mechanism will develop down to scales of $100 \mathrm{~km}$ or less. This assumption is consistent with the observations from the Atmospheric Imaging Assembly (AIA), the EUV telescope on board the recently launched Solar Dynamics Observatory (SDO) mission [11]. AIA images the Sun at a four times better resolution than EIT. Multiscaling behaviors computed on AIA images are even clearer than and consistent with previous observations from EIT. Compared to other works, our method is more flexible since it adds details at higher resolution in a stochastic manner. However a fundamental assumption but also limitation of our procedure is that the scale invariance properties of the original image must be known. This is the case for synthetic textures, or for images that have been previously analyzed. 


\subsection{Fractionally integrated compound Poisson cascades}

Our approach relies on a model that reproduces the multifractal properties of quiet Sun images. It is described by:

$$
I_{1}=I_{0}+\alpha \mathcal{I}_{H}\left\{Q_{r_{1}}^{r_{0}}-\left\langle Q_{r_{1}}^{r_{0}}\right\rangle\right\}
$$

where $I_{0}$ stands for the average intensity of the image, \langle\rangle is spatial averaging; $\alpha>0$ is an amplification factor; $\mathcal{I}_{H}$ is the fractional integration (FI) performed by using a $1 /\|k\|^{H}$ low-pass filter in the Fourier domain and $Q_{r_{1}}^{r_{0}}$ is a compound Poisson cascade (CPC). The scales $r_{0}$ and $r_{1}$ stand for the largest scale and the finest scale (the pixel size) of the image. CPC $[12,13]$ are a family of multifractal measures that can be easily simulated with prescribed multifractal exponents $\tau(q)$. Then the fractional integration yields the field $I$ characterized by $\zeta(q)=q H+\tau(q)$. See $[2,4]$ for a detailed presentation.

\subsection{Validation of the model}

We have proposed a parametric model of the form given by eq. (2) leading to

$$
\zeta(q)=\left\{\begin{array}{lll}
q H+1-\frac{(1+T)^{q}}{(1+q T)} & \text { for } \quad 0 \leq q \leq q_{+}^{*}, \\
2-q \zeta^{\prime}\left(q_{+}^{*}\right) & \text { for } \quad q \geq q_{+}^{*},
\end{array}\right.
$$

where $H \simeq 0.6, q_{+}^{*} \simeq 2.25$ and $T=0.85$ [2]. A quantitative validation of this model is provided by a multifractal analysis which yields the same multiscaling exponents for both the model and the data. The rather satisfying visual aspect of the synthetic images compared to observations provides a qualitative confirmation, fig. 1. This illustrates the strong link between the statistical properties of an image and its aspect. Taking the multifractal properties into account is crucial.

\section{VIRTUAL SUPER-RESOLUTION}

\subsection{Method}

We now aim at coupling the data with the model to propose a plausible prediction of what the unavailable high resolution information could look like. Such a prediction must obey both the statistical and physical consistency evoked above. Based on the model of eq. (2), this is made possible thanks to the following essential property of CPC. For any $0<r_{2} \leq r_{1} \leq$ $r_{0}, Q_{r_{2}}^{r_{0}}$ obeys a multiplicative multiscaling decomposition

$$
Q_{r_{2}}^{r_{0}}=Q_{r_{1}}^{r_{0}} \cdot Q_{r_{2}}^{r_{1}}
$$

where $Q_{r_{1}}^{r_{0}}$ and $Q_{r_{2}}^{r_{1}}$ are two independent CPC with the same multifractal exponents $\tau(q)$. The main thrust is to replace $Q_{r_{1}}^{r_{0}}$ by $Q_{r_{2}}^{r_{0}}$ in (2) to get $I_{2}$ at resolution $r_{2}<r_{1}$, see [4] for details. Eventually, a renormalization step ensures that the procedure is conservative in the sense that zooming out to the initial resolution gives the original image back. We checked numerically that this last step does not affect the scale invariance properties of the resulting image.

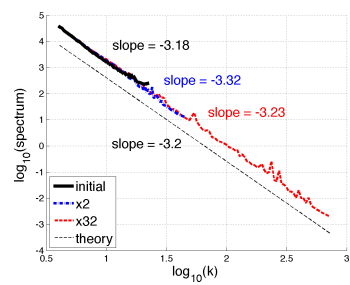

(a)

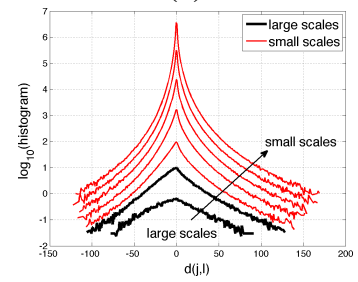

(c)

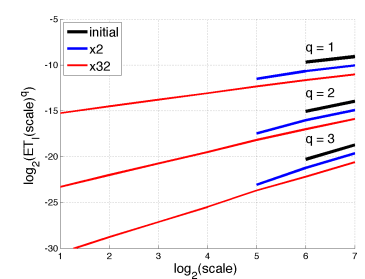

(b)

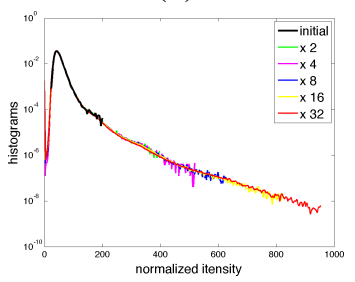

(d)
Fig. 2. (a) Power law Fourier spectra; (b) multiscaling at order 1,2 and 3 of magnified images $\times 2$ and $\times 32$; (c) histograms of wavelet coefficients for various scales from original (black) and augmented (red) images ; (d) intensity histograms for various super-resolution factors.

\subsection{Results \& illustration}

We have checked that the super-resolved images both consistently extrapolate multifractal properties in the scale domain and visually and physically consistently add new details in the space domain. Fig. 2 shows the extrapolation of the powerlaw Fourier spectrum and multiscaling behaviors as well as the evolution of wavelet coefficients histograms towards less and less Gaussian distributions, which is typical of multifractal images.

\section{APPLICATIONS \& PERSPECTIVES}

\subsection{Prediction of intensity histograms}

An interesting prediction of our approach is the extrapolation of the intensity histogram of high resolution images, fig. 2(d). In the multifractal framework, the usual intuition is that smaller scales contribute to higher and higher departures from Gaussian distributions. Therefore one may expect that super-resolved images have heavier tailed histograms than low-resolution images. However this intuition is correct for sufficiently irregular images only. For the model (2) fitted to EIT data with $H \simeq 0.6>1 / 2$, the histogram of superresolved images is almost identical to low-resolution images [4]. This can be checked by simulating enough low-resolution images to estimate statistics from equivalent amounts of data for low and high resolution images (the latter contains many more pixels). The same experiment applied to the choice $H<1 / 2$ yields different results in agreement with the usual multifractal based intuition. Thus, usual arguments valid for 
regular images can be used to predict the exposure level of multifractal but smooth enough images $(H>1 / 2)$ at high resolution from low resolution images.

\subsection{Denoising of quiet Sun images}

The photon emission processes in the solar atmosphere are random and incoherent in nature. They are usually modeled by a Poisson process, denoted by $\mathcal{P}($.$) below. Images$ recorded by EIT or AIA are coming through similar acquisition systems. Those yield a signal $s=\beta \mathcal{P}\left(\gamma \times \operatorname{PSF} * \phi_{0}\right)$ where $\phi_{0}$ is the photon emission process, and $\beta>0$ and $\gamma>0$ are amplification factors; ' $*$ ' is convolution. The synthetic images are exempt of noise and may serve as a reference. For instance, the the little flattening of the Fourier spectrum and structure functions at small scales (high frequencies) may a priori be due to either the PSF of the sensor or the presence of noise. We can show it is in fact due to noise. At EIT resolution, there are enough photons per pixel so that Poisson noise is very similar to Gaussian noise. We have tried several state of the art Poisson denoising methods. A simple Wiener filter based on the prior of a power-law Fourier spectrum seems to work very well. Indeed it preserves small scale details more accurately than many Poisson denoising methods which use some regularization principle that generally slightly over-smoothes the image.

\subsection{Calibration of a stereo reconstruction algorithm}

The model eq. (2) can be adapted on the sphere [5]. The mission STEREO launched in 2006 has provided astronomers with images from 2 synchronized spatial telescopes, allowing for the 3D reconstruction of the Sun. The algorithm described in [14] allows for a 3D reconstruction of solar coronal structures. Its accuracy is difficult to quantify since its performance depends on the textural aspect of the images. Thanks to our model, a 3D spherical model of the Sun with realistic texture and altitude variations can be built which allows for the 3D algorithm benchmarking (bias, variance). This ongoing work is essential to validate the detection of special events such as filaments which typically reach altitudes of about $5 \%$ of the Sun radius before erupting into space.

\section{CONCLUSION}

We have presented the whole trajectory of a work on the analysis, modeling and virtual super-resolution of quiet Sun images provided by the spatial telescope EIT in the extreme UV. The first step dealt with the validation of scale invariance and the wavelet based multifractal analysis of these images so as to estimate their characteristic multiscaling exponents $\zeta(q)$. Then we have proposed a fractionally integrated compound Poisson cascade (FI-CPC) as a constructive parametric model that reproduces the scale invariance properties of quiet Sun images. Virtual super-resolution is made possible by coupling present low-resolution images with the model to add statistically and physically plausible subpixel details. Most of all this approach extrapolates the scale invariance properties of the initial image. Such an approach can be useful to various respects such as to one day decide upon consistency with respect to the extrapolated behaviors. Perspectives include the search for a maximum likelihood super-resolution or the extension to 3D atmospheric cloud field models.

\section{REFERENCES}

[1] E. R. Priest, J. F. Heyvaerts, and A. M. Title, "A Flux-Tube Tectonics Model for Solar Coronal Heating Driven by the Magnetic Carpet," The Astrophys. J., vol. 576, pp. 533-551, 2002.

[2] V. Delouille, P. Chainais, and J.-F. Hochedez, "Quantifying and containing the curse of high resolution coronal imaging," Annales Geophysicae, vol. 26, no. 10, pp. 3169-3184, 2008.

[3] P. Kestener et al., "Characterising complexity in solar magnetogram data using a wavelet-based segmentation method," The Astrophys. J., vol. 717, no. 2, pp. 995, 2010.

[4] P. Chainais, E. Kœnig, V. Delouille, and J.-F. Hochedez, "Virtual super resolution of scale invariant textured images using multifractal stochastic processes," Journal of Mathematical Imaging and Vision, vol. 39, no. 1, pp. 28-44, 2011.

[5] E. Kœnig and P. Chainais, "Multifractal analysis on the sphere," in Image and Signal Processing, A. Elmoataz et al., Ed., vol. 5099 of L.N.C.S., pp. 613-621. Springer, 2008.

[6] J.-P. Delaboudinière and the EIT Team, "EIT: ExtremeUltraviolet Imaging Telescope for the SOHO Mission," Solar Physics, vol. 162, pp. 291-312, 1995.

[7] D. Berghmans, F. Clette, and D. Moses, "Quiet Sun EUV transient brightenings and turbulence. A panoramic view by EIT on board SOHO," Astronomy and Astrophysics, vol. 336, pp. 1039-1055, Aug. 1998.

[8] M. J. Aschwanden and C. E. Parnell, "Nanoflare Statistics from First Principles: Fractal Geometry and Temperature Synthesis," The Astrophys. J., vol. 572, pp. 1048-1071, June 2002.

[9] A. Srivastava, A.B. Lee, E.P. Simoncelli, and S.-C. Zhu, "On advances in statistical modeling of natural images," Journal of Mathematical Imaging and Vision, vol. 18, pp. 17-33, 2003.

[10] S. Jaffard, "Multifractal formalism for functions, part $1 \& 2$," SIAM J. of Math. Anal., vol. 28, no. 4, pp. 944-998, 1997.

[11] “Solar Dynamics Observatory," http://sdo.gsfc.nasa.gov/data/.

[12] J. Barral and B. Mandelbrot, "Multiplicative products of cylindrical pulses," Proba. Theory \& Relat. Fields, vol. 124, pp. 409-430, 2002.

[13] P. Chainais, "Multidimensional infinitely divisible cascades. Application to the modelling of intermittency in turbulence," Eur. J. Phys. B, vol. 51, pp. 229-243, 2006.

[14] S. F. Gissot, J.-F. Hochedez, P. Chainais, and J.-P. Antoine, "3D reconstruction from SECCHI-EUVI images using an opticalflow algorithm: Method description and observation of an erupting filament," Solar Physics, vol. 252, no. 2, pp. 397408, 2008. 